\title{
Diurnal Fluctuation of Sleep Propensity and Hormonal Secretion across the Menstrual Cycle
}

\author{
Kayo Shibui, Makoto Uchiyama, Masako Okawa, Yoshihisa Kudo, Keiko Kim, \\ Xianchen Liu, Yuichi Kamei, Tatsuro Hayakawa, Tatsuya Akamatsu, Katsuya Ohta, \\ and Kenichi Ishibashi
}

Background: The fact that most women experience sleep changes across the menstrual cycle is thought to be associated with changes in circadian rhythms; however, few studies have investigated this relationship.

Methods: We applied an ultrashort sleep-wake schedule to eight healthy women and studied diurnal fluctuations in sleep propensity, sleepiness, rectal temperature, and serum concentrations of melatonin, thyroid-stimulating hormone, and cortisol in the follicular and luteal phases.

Results: In the luteal phase, amplitude of core body temperature, total melatonin secretions, and amplitudes of TSH and cortisol rhythms were significantly decreased, whereas sleepiness and occurrence of slow-wave sleep during the daytime were significantly increased. Differences in the amount of daytime slow-wave sleep across the menstrual cycle were positively correlated with differences in the daily mean rectal temperature.

Conclusions: The findings suggest that the amplitude of circadian oscillation may be dampened in the luteal phase. Increased daytime sleepiness in the luteal phase may be associated with increased daytime slow-wave sleep, due possibly to changes in thermoregulation in the luteal phase. Biol Psychiatry 2000;48:1062-1068 (C) 2000 Society of Biological Psychiatry

Key Words: Circadian rhythm, core body temperature, hormonal rhythm, melatonin, menstrual cycle, sleep

From the Department of Psychophysiology, National Institute of Mental Health (KS, MU, YK, KK, XL) and the Department of Psychiatry, Kohnodai Hospital, National Center of Neurology and Psychiatry (YK, TH), Ichikawa; the Department of Psychiatry, Shiga University of Medical Science, Shiga (MO); the Department of Obstetrics and Gynecology, Showa University School of Medicine (TA) and the Department of Neuropsychiatry, Tokyo Medical and Dental University School of Medicine (KS, KO), Tokyo; and the Department of Psychiatry and Neurosciences, Hiroshima University School of Medicine, Hiroshima (KI), Japan.

Address reprint requests to Makoto Uchiyama, M.D., Ph.D., Department of Psychophysiology, National Institute of Mental Health, NCNP, 1-7-3 Kohnodai, Ichikawa, Chiba 272-0827, Japan.

Received August 16, 1999; revised December 7, 1999; revised March 27, 2000; accepted April 28, 2000

\section{Introduction}

S leep changes across the menstrual cycle are commonly Nexperienced by normal ovulatory women (Clare 1983; Mauri et al 1988; Sheldrake and Cormack 1976). Although there are several hypotheses on possible physiologic backgrounds underlying these sleep changes across the menstrual cycle, the pathophysiologic mechanism still remains unclear.

Evidence of differences in sleep architecture between the follicular and luteal phases (FP and LP) has been demonstrated by all-night polysomnography in previous studies (Ito et al 1995; Parry et al 1989). Parry et al (1989) found that stage 3 sleep and intermittent awakening varied with phases of the menstrual cycle. Ito et al (1995) reported that the amount of slow-wave sleep (SWS) decreased significantly in the LP, as compared with the FP, in healthy women; however, other studies (Chuong et al 1997; Driver et al 1996) reported no significant difference in sleep architecture between the FP and the LP. The inconsistent data on sleep changes across the menstrual cycle in previous studies may be explained by the effects of environmental factors, social activities, and the subjects involved. Therefore, stricter controls of diurnal behavior and environmental factors may be needed for polysomnographic studies examining changes in sleep architecture across the menstrual cycle.

Circadian aspects of sleep changes across the menstrual cycle in healthy women have been investigated. Lee (1988) demonstrated that the amplitude of core body temperature rhythm in the LP was dampened, as compared with the FP. Rogacz et al (1987) reported that the temperature rhythm was phase delayed in the LP. Webley and Leidenberger (1986) found that the amount of daily melatonin production was significantly increased in the LP; however, several other studies (Berga and Yen 1990; Kivelä et al 1988; Parry et al 1990) found no such changes. These inconsistent findings may be explained by the influence of such noncircadian factors as ambient illumination or behavioral factors, both of which can obscure or mask the underlying oscillatory components of those 
rhythms (Barrett et al 1993; Brainard et al 1988; Carrier and Monk 1997; Minors and Waterhouse 1989).

To exclude the masking effects of light and behavior on circadian rhythms and to measure outputs of the circadian pacemaker, we conducted multiple nap tests using the ultradian routine method (Lack and Lushington 1996; Lavie 1986) together with simultaneous measurement of hormonal rhythms under dim light conditions. Our particular interest was to clarify the diurnal fluctuations of sleep propensity (SP), core body temperature, and serum hormonal concentrations.

\section{Methods and Materials}

\section{Subjects}

Eleven healthy women, aged 20 to 23 years, participated in the study as paid volunteers after giving their informed consent. They had no history of major medical, gynecologic, or psychiatric illness or family history of psychiatric illness. They had not taken oral contraceptives and had not been pregnant. For screening, we instructed all of the subjects to record basal body temperature (BBT) data, a five-point mood scale, and a sleep log daily for more than one menstrual cycle. During the last 7-10 days of the screening period, subjects wore a wrist activity recorder (Actigraph, AMI, New York). The sleep log data together with actigraphy confirmed that they were good sleepers with regular sleep-wake schedules. A gynecologic specialist (TA) blindly inspected BBT records of the subjects. Three subjects out of 11 were excluded because their BBT records did not show apparent biphasic pattern across the menstrual cycle. Worsening of mood in LP was not detected in any of the subjects. Psychiatric interviews revealed that their menstrual-associated complaints did not meet the criteria of premenstrual tension syndrome (Reid 1985) or of premenstrual dysphoric disorder (American Psychiatric Association 1994). No subjects were excluded by psychiatric problems. Finally, eight participants, who had regular cycles of 28 to 32 days with an apparent biphasic pattern of BBT fluctuation across the cycle, entered the protocol. All of them completed the study.

\section{Experimental Procedures}

A 3-day experimental laboratory session with a crossover design was conducted in the FP and the LP. The FP session started on days 5 to 8 after menses onset, and the LP session started on days 3 to 5 before menses onset. The sleep schedule for 5 days preceding each experimental session was validated by sleep logs and actigraphic data. Each subject was instructed to wake up at 8:00 AM on day 1 of each session and come to the laboratory in the evening. They were kept awake in a sitting position under constant room light $(<50$ lux $)$ wearing a rectal temperature apparatus (resolution $0.01^{\circ} \mathrm{C}$, Kohden Medical, Tokyo) and activity recorders. At 8:00 AM on the second day the subjects underwent the ultrashort sleep-wake schedule for 26 hours. In each 30-min period they had a 10-min nap trial in darkness $(<1$ lux); they were allowed to sleep, and standard polysomnographs were recorded. During the 20-min periods between consecutive nap trials, they were kept awake in a sitting position under dim light ( $<10$ lux). When leaving the room for urination/defecation, subjects wore dark goggles that attenuated light coming into the eyes to less than 10 lux. Subjective sleepiness was assessed according to the Stanford Sleepiness Scale (SSS; Hoddes et al 1973) every $30 \mathrm{~min}$. Subjects were occupied with activities such as reading, watching videos, playing cards, and casual conversation. The room temperature was maintained between $23^{\circ} \mathrm{C}$ and $25^{\circ} \mathrm{C}$. The subjects took $630-\mathrm{kJ}$ isocaloric snacks and 100 to 150 $\mathrm{mL}$ of water every 2 hours. Blood samples were collected through indwelling intravenous catheters with a heparin lock every hour. Having been centrifuged, serum was stored at $-70^{\circ} \mathrm{C}$.

HORMONAL ASSAYS. Serum melatonin levels were assayed by radioimmunoassay (Bühlmann Melatonin Radioimmunoassay Test Kit, Allshwil, Switzerland), with a sensitivity of 0.3 $\mathrm{pg} / \mathrm{mL}$. Serum levels of thyroid-stimulating hormone (TSH), estradiol, progesterone, and cortisol were analyzed by radioimmunoassay.

ASSESSMENT OF SUBJECTIVE SLEEPINESS. We analyzed 48 SSS scores for 24 hours, excluding the first and last hours. The mean SSS scores for each hour were used for statistical analysis. To compare the trend of subjective sleepiness across the day, we grouped the data obtained in the 24 hours into three periods: 9:00 AM to 4:30 PM (daytime), 5:00 PM to 12:30 AM (late evening), and 1:00 AM to 8:30 AM (nighttime). The third 8-hour period was expected to correspond to significant melatonin production $(>50 \%$ of the peak). The first and the second 8-hour periods were supposed to correspond to hours in which melatonin secretion was unlikely.

ASSESSMENT OF SLEEP EEG. We analyzed 48 nap trials from 24 hours, excluding the first and last two nap trials. Polysomnographic recordings were scored according to standard criteria (Rechtschaffen and Kales 1968). The SP for each 30-min cycle was defined as the sum of the duration of stages 2,3 , and 4 sleep and rapid eye movement sleep. The mean SP for every hour was used for statistical analysis. To compare the trend of sleep variables across the day, we grouped the data obtained in the 24 hours into three periods as described above.

ASSESSMENT OF RECTAL TEMPERATURE AND HORMONAL RHYTHMS. The rectal temperature curves (24 hours) for each session were fitted with complex cosine curves (24-hour plus 12-hour rhythms) to determine the maxima and minima. The amplitude of the rectal temperature rhythm was defined as half of the difference between the maxima and minima. The daily mean rectal temperature was calculated by averaging the 5-min data for 24 hours. The mean rectal temperatures for each hour were plotted and used for statistical analysis.

Single cosinor analysis was applied to fit melatonin, TSH, and cortisol rhythms to obtain acrophase and amplitude. The melatonin onset and offset times were determined visually as the crossing times of the raw data and the midrange of peak 


\section{Follicular Phase}
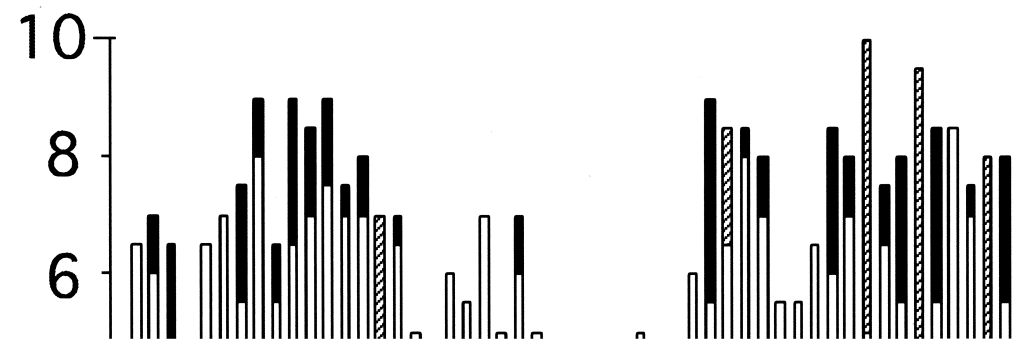

Figure 1. Sleep propensity in the follicular and luteal phases in a representative subject. The fluctuation of sleep propensity had two components: a daytime rise and a nighttime rise in the evening in both the follicular and the luteal phase. Naps containing slow-wave sleep in the daytime were more frequent in the luteal phase than in the follicular phase. There were no marked changes in nighttime sleep propensity between the two menstrual phases. White bar, stage 2 sleep; black bar, stages 3-4 sleep; shaded bar, rapid eye movement sleep.

concentration. The melatonin duration was defined as the period between melatonin onset and offset times. The level of melatonin was analyzed as absolute values and area under the curve (AUC). The latter was calculated by hourly integrated values for 24 hours.

\section{Statistical Analysis}

Hourly data obtained were submitted to two-way analysis of variance (ANOVA) for repeated measures with a correction of Greenhouse-Geisser epsilon. The Wilcoxon signed rank test was used for two group analyses between the FP and the LP. We calculated the differences $(\Delta)$ between the FP and the LP in parameters concerning sleep, core body temperature, and hormonal rhythms. The Spearman rank correlation coefficient was used to assess association between pairs of given measures. Differences at $p<.05$ were accepted as statistically significant. All statistical analyses were conducted using the SYSTAT statistical program (SYSTAT, Chicago).

\section{Results}

\section{Overview}

Sleep propensity data obtained from a representative subject are shown in Figure 1. For this subject, the fluctuation of SP had two components: a daytime rise and a nighttime rise in both the FP and the LP. Naps containing SWS in the daytime were more frequent in the LP than in 
Table 1. Comparison of the Number of Naps Containing Slow-wave Sleep and Stanford Sleepiness Scale Scores between Follicular and Luteal Phases

\begin{tabular}{lccc}
\hline & $\begin{array}{c}\text { Follicular } \\
\text { phase }\end{array}$ & $\begin{array}{c}\text { Luteal } \\
\text { phase }\end{array}$ & $p$ \\
\hline $\begin{array}{l}\text { Number of naps containing slow } \\
\text { wave sleep (n) }\end{array}$ & & & \\
9:00 AM-4:30 PM & $3.5 \pm 1.2$ & $5.9 \pm 1.2$ & $<.05$ \\
5:00 PM-12:30 AM & $2.1 \pm 0.6$ & $2.5 \pm 1.0$ & $\mathrm{~ns}$ \\
1:00 AM-8:30 AM & $6.0 \pm 1.2$ & $6.0 \pm 1.0$ & $\mathrm{~ns}$ \\
Stanford Sleepiness Scale scores & & & \\
9:00 AM-4:30 PM & $3.6 \pm 0.2$ & $4.0 \pm 0.1$ & $<.05$ \\
5:00 PM-12:30 AM & $3.5 \pm 0.2$ & $3.7 \pm 0.1$ & $\mathrm{~ns}$ \\
1:00 AM-8:30 AM & $5.1 \pm 0.2$ & $5.2 \pm 0.1$ & $\mathrm{~ns}$ \\
\hline
\end{tabular}

Each value is shown as mean $\pm \mathrm{SE}$

the FP. There were no marked changes in nighttime SP between the two menstrual phases. Similar findings were observed for the other subjects.

\section{Sleep Propensity Measures}

Repeated two-way ANOVA revealed a significant effect of time course on SP $[\epsilon(23)=.18, p<.001]$, whereas the menstrual phase had no significant effect. In the daytime (9:00 AM-4:30 PM), the number of SWS-containing naps was significantly increased in the LP from that in the FP $(5.9 \pm 1.2$ vs. $3.5 \pm 1.2, p<.05$, Wilcoxon signed rank test). In the late evening and nighttime (5:00 $\mathrm{PM}-12: 30 \mathrm{AM}$ and 1:00 AM-8:30 AM), the number of SWS-containing naps did not differ in the FP and the LP (Table 1).

\section{Subjective Sleepiness}

Time course had a significant effect on SSS scores $[\epsilon(23)=.13, p<.01]$, whereas the menstrual phase had no significant effect. In the daytime (9:00 AM-4:30 PM), the mean SSS scores were significantly higher in the LP $(4.0 \pm 0.1$ vs. $3.6 \pm 0.2, p<.05$, Wilcoxon signed rank test). In the late evening and nighttime (5:00 PM-12:30 AM and 1:00 AM-8:30 AM), the mean SSS scores did not differ in the FP and the LP (Table 1).

Pre-Experimental Sleep, Temperature Rhythms, and Hormonal Parameters

Actigraphic data showed that the sleep duration for the 5 days preceding the experimental session did not differ in the two menstrual phases $(7.00 \pm 0.26$ hours in the FP vs. $7.22 \pm 0.22$ hours in the LP).

Figure 2 illustrates 24-hour profiles of core body temperature, melatonin, TSH, cortisol, estradiol, and progesterone measured during the FP and the LP for the eight subjects.

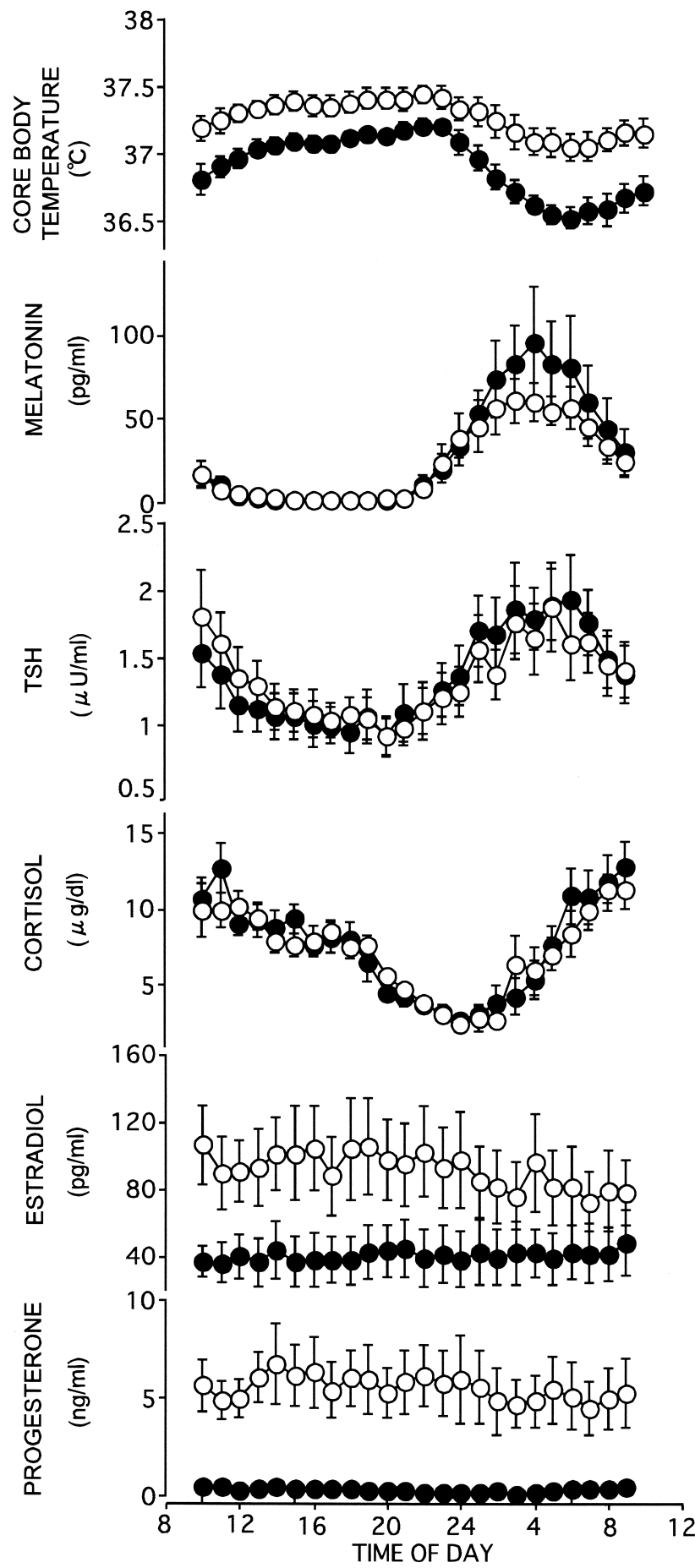

Figure 2. Twenty-four-hour profiles of core body temperature, melatonin, thyroid-stimulating hormone (TSH), cortisol, estradiol, and progesterone measured during the follicular and luteal phases (FP and LP) for eight subjects in mean and SE. The plots represent the values for the FP $(O)$ and the LP $(\bigcirc)$.

For mean core body temperature, two-way repeated ANOVA revealed that time course $[\epsilon(23)=.11, p<$ $.001]$, menstrual phase ( $\mathrm{df}=1, p<.001)$, and their 
Table 2. Circadian Parameters of Core Body Temperature, Melatonin, TSH, and Cortisol in Eight Women during the Follicular and Luteal Phases

\begin{tabular}{lccc}
\hline Parameters & Follicular phase & Luteal phase & $p$ \\
\hline Core body temperature & & & \\
$\quad$ Nadir & $5: 51 \mathrm{AM} \pm 0: 20$ & $5: 40 \mathrm{AM} \pm 0: 20$ & $\mathrm{~ns}$ \\
Minima $\left({ }^{\circ} \mathrm{C}\right)$ & $36.48 \pm 0.08$ & $37.03 \pm 0.11$ & $<.0001$ \\
Maxima $\left({ }^{\circ} \mathrm{C}\right)$ & $37.24 \pm 0.03$ & $37.45 \pm 0.07$ & $<.01$ \\
Mean $\left({ }^{\circ} \mathrm{C}\right)$ & $36.93 \pm 0.03$ & $37.28 \pm 0.08$ & $<.05$ \\
Amplitude $\left({ }^{\circ} \mathrm{C}\right)$ & $0.38 \pm 0.03$ & $0.20 \pm 0.03$ & $<.0001$ \\
Melatonin & & & \\
AUC $(24$ hour$)$ & $736.16 \pm 199.34$ & $573.86 \pm 120.07$ & $<.05$ \\
Maxima $(\mathrm{pg} / \mathrm{mL})$ & $102.25 \pm 31.44$ & $75.13 \pm 14.65$ & $\mathrm{~ns}$ \\
Acrophase & $3: 56 \mathrm{AM} \pm 0: 38$ & $4: 00 \mathrm{AM} \pm 0: 40$ & $\mathrm{~ns}$ \\
Onset time & $12: 07 \mathrm{AM} \pm 0: 41$ & $12: 07 \mathrm{AM} \pm 0: 44$ & $\mathrm{~ns}$ \\
Offset time & $7: 39 \mathrm{AM} \pm 0: 38$ & $7: 38 \mathrm{AM} \pm 0: 41$ & $\mathrm{~ns}$ \\
Duration $($ hours $)$ & $7.53 \pm 0.45$ & $7.51 \pm 0.37$ & $\mathrm{~ns}$ \\
TSH & & & \\
Mean $(\mu \mathrm{U} / \mathrm{mL})$ & $1.37 \pm 0.20$ & $1.36 \pm 0.20$ & $\mathrm{~ns}$ \\
Acrophase & $4: 58 \mathrm{AM} \pm 0: 38$ & $6: 20 \mathrm{AM} \pm 0: 19$ & $<.05$ \\
Amplitude $(\mu \mathrm{U} / \mathrm{mL})$ & $0.50 \pm 0.09$ & $0.37 \pm 0.08$ & $<.05$ \\
Cortisol & & & \\
Mean $(\mu \mathrm{g} / \mathrm{dL})$ & $7.54 \pm 0.39$ & $7.36 \pm 0.38$ & $\mathrm{~ns}$ \\
Acrophase & $10: 48 \mathrm{AM} \pm 0: 47$ & $10: 41 \mathrm{AM} \pm 0: 40$ & $\mathrm{~ns}$ \\
Amplitude $(\mu \mathrm{g} / \mathrm{dL})$ & $4.78 \pm 0.23$ & $3.95 \pm 0.35$ & $<.05$ \\
\hline
\end{tabular}

Values are means \pm SEs. TSH, thyroid-stimulating hormone; AUC, area under the curve.

interaction $[\epsilon(23)=.16, p<.01]$ were significant. As shown in Figure 2, the difference of core body temperature between the FP and the LP was greater at night. The Wilcoxon signed rank test revealed that individual daily mean core body temperature was significantly elevated in the LP $(p<.0001)$, whereas the amplitude was significantly decreased $(p<.0001)$. The nadir of the core body temperature curve did not differ in the FP and the LP (Table 2).

For melatonin secretion, we found a significant effect only for time course $[\epsilon(23)=.07, p<.01]$. As shown in Figure 2, plasma melatonin concentrations were higher during the night. Comparison of individual chronobiological measurements of melatonin secretion indicated that the onset time, offset time, acrophase, duration, and maxima of melatonin rhythm did not differ in the FP and the LP. The 24-hour AUCs of melatonin were smaller in the LP $(p<.05$; Table 2).

For TSH secretion, we found a significant effect for time course $[\epsilon(23)=.12, p<.001]$. As shown in Figure 2 , TSH showed significant circadian rhythms, with higher values during the night. When individual chronobiological measurements of TSH secretion were compared between the FP and the LP, the mean concentrations of TSH did not differ significantly. The acrophase of TSH was significantly delayed in the LP $(p<.05)$, and its amplitude was smaller $(p<.05$; Table 2).

For cortisol, time course also had a significant effect $[\epsilon(23)=.12, p<.001]$. As illustrated in Figure 2, cortisol showed significant circadian rhythms, with higher values in the early morning. When individual chronobiological measurements of cortisol rhythm were compared between the FP and the LP, the mean value and the acrophase of the cortisol curve did not differ significantly, but its amplitude was smaller in the LP $(p<.05$; Table 2).

For estradiol, repeated two-way ANOVA revealed an interaction between time course and menstrual phase $[\epsilon(23)=.15, p<.05]$. As shown in Figure 2, the differences in estradiol levels between the FP and the LP were larger in the daytime. When individual levels of estradiol were compared between the FP and the LP, mean estradiol level had no significant difference.

For progesterone, menstrual phase had a significant effect $(\mathrm{df}=1, p<.01)$. As shown in Figure 2, progesterone levels were markedly different in the FP and the LP, with higher values in the LP (LP, $5.54 \pm 1.49$ $\mathrm{ng} / \mathrm{mL}$, vs. FP, $0.35 \pm 0.06 \mathrm{ng} / \mathrm{mL}$ ).

\section{Correlation between $\Delta$ SWS and Core Body Temperature/Hormone Parameters}

Spearman rank correlation coefficients were calculated to show the associations of $\Delta$ SWS parameters (amount and number) with $\Delta$ daily mean core body temperature/ $\Delta$ amplitude of core body temperature rhythm $/ \Delta$ AUC of melatonin/ $\Delta$ daily mean TSH concentrations $/ \Delta$ amplitude of TSH rhythm/ $\Delta$ daily mean cortisol concentrations $/ \Delta$ amplitude of cortisol rhythm/ $\Delta$ daily mean progesterone concentrations/ $\Delta$ daily mean estradiol concentrations. We found that only $\Delta$ amount of SWS in the daytime (9:00 AM-4:30 PM) had a significantly positive correlation with $\Delta$ daily mean core body temperature $(\rho=.78, p<.05)$.

\section{Discussion}

The fluctuations of subjective sleepiness across the menstrual cycle, together with those of SP, rectal temperature, and serum hormonal concentrations, were investigated using an ultrashort sleep-wake schedule. Results indicated that amplitude of rectal temperature, total melatonin secretions, and amplitudes of TSH and cortisol rhythms were significantly decreased in the LP, whereas sleepiness and occurrence of SWS during the daytime were significantly increased. Furthermore, the differences in the amount of daytime SWS across the menstrual cycle were positively correlated with the differences in the daily mean core body temperature.

Previous studies demonstrated that subjective daytime sleepiness was more frequent in the premenstrual period (Clare 1983; Sheldrake and Cormack 1976). To our 
knowledge, no investigations have applied objective methods to examine daytime sleepiness across the menstrual cycle. Here, we studied diurnal fluctuations in subjective sleepiness, together with SP in the FP and the LP. In the LP, subjective sleepiness and occurrence of SWS during the daytime were significantly increased.

A prior nocturnal polysomnographic study revealed that stage 3 sleep and intermittent awakenings varied with phases of the menstrual cycle (Parry et al 1989). More recently, Ito et al (1995) reported that SWS was decreased in the LP, as compared with the FP. The results of previous studies on the changes in nocturnal sleep across the menstrual cycle may have been confounded by changes of daytime activity and/or environmental factors across the menstrual cycle. To minimize these confounding factors, we measured SP rhythm for 24 hours under well-controlled conditions. An ultrashort sleep-wake schedule has been used to study diurnal fluctuation of SP (Lack and Lushington 1996; Lavie 1986; Nakagawa et al 1992). In our study SP rhythm consisted of two components in both the FP and the LP: a daytime rise of SP likely to correspond to recovery sleep due to 24-hour sleep deprivation, and an increasing nighttime rise of SP in parallel with melatonin rise. During the daytime (9:00 AM-4:30 PM), both subjective sleepiness and the number of SWS-containing naps significantly increased in the LP; however, in the other two periods (5:00 PM-12:30 AM and 1:00 AM-8:30 AM) subjective sleepiness and the number of SWS-containing naps did not differ in the two conditions. Neither pre-experimental sleep conditions nor sleep variables during the night differed significantly. The increased number of SWS-containing naps during the daytime suggests that demand for daytime SWS might have increased in the LP regardless of nocturnal sleep. Hormonal changes in the LP might have affected a compensatory function for sleep loss.

In our study the 24-hour AUC of melatonin was significantly smaller in the LP than in the FP. In the previous studies, data on differences in melatonin secretion in healthy women across the menstrual cycle were inconsistent. Webley and Leidenberger (1986) showed that melatonin AUC was greater in the LP; however, several reports (Berga and Yen 1990; Kivelä et al 1988; Parry et al 1990) showed that melatonin levels were not significantly different across the menstrual cycle. In the present study, blood samples were obtained under constant dim light conditions $(<10$ lux ) for 26 hours. Under dim light conditions, the melatonin rhythm is thought to represent the oscillation of the circadian pacemaker (Lewy and Sack 1988). Inconsistent results obtained in the previous studies may have been confounded by different light conditions. Our findings of reduced melatonin secretion, together with reduced amplitudes of core body temperature, cortisol, and TSH, seemed to be accounted for by a reduction in the amplitude of the endogenous oscillation of the circadian pacemaker (Rodier 1971) instead of the direct effect of progesterone on hormonal secretion, since orally administered progesterone seemed to increase the serum melatonin level (Webley and Leidenberger 1986).

In this study the acrophases of temperature, melatonin, and cortisol rhythms did not differ significantly between the FP and the LP, whereas the acrophase of TSH was phase delayed significantly in the LP. Endogenous TSH rhythm is governed by the circadian pacemaker that drives many other circadian rhythms (Allan and Czeisler 1994), but TSH secretion is known to be modified by the thermoregulation system in the brain (Fisher and Odell 1971). Our finding of delayed TSH rhythms in the LP may be related to the high core body temperature in the LP. Another possible explanation is based on the ability of progesterone to phase-delay circadian oscillation in animals (Axelson et al 1981); however, this assumption is unlikely because other rhythms were not significantly phase delayed.

We found that $\Delta$ amount of SWS in the daytime (9:00 AM-4:30 PM) was positively correlated with $\Delta$ mean daily core body temperature. Given that there was a strong correlation between the increased SWS and the elevation of core body temperature (Berger et al 1988), the SWS regulatory system and thermoregulatory system may be changed across the menstrual cycle.

Because the small number of subjects involved in this study may limit our conclusions, further studies with a larger number of subjects are needed to confirm our findings.

\section{References}

Allan JS, Czeisler CA (1994): Persistence of circadian thyrotropin rhythm under constant conditions and after lightinduced shifts of circadian phase. J Clin Endocrinol Metab 79:508-512.

American Psychiatric Association (1994): Diagnostic and Statistical Manual of Mental Disorders, 4th ed. Washington, DC: American Psychiatric Association Press.

Axelson JF, Gerall AA, Albers HE (1981): Effect of progesterone on the estrous activity cycle of rat. Physiol Behav 26:631-635.

Barrett J, Lack L, Morris M (1993): The sleep-evoked decrease of body temperature. Sleep 16:93-99.

Berga SL, Yen SSC (1990): Circadian pattern of plasma melatonin concentrations during four phases of the human menstrual cycle. Metabolism 51:1606-1612.

Berger RJ, Palca JW, Walker JM, Phillips NH (1988): Correlations between body temperatures, metabolic rate and slow wave sleep in humans. Neurosci Lett 86:230-234.

Brainard GC, Lewy AJ, Menaker M, Fredrickson RH, Miller LS, 
Weleber RG, et al (1988): Dose-response relationship between light irradiance and the suppression of plasma melatonin in human volunteers. Brain Res 454:212-218.

Carrier J, Monk TH (1997): Estimating the endogenous circadian temperature rhythm without keeping people awake. J Biol Rhythms 12:266-277.

Chuong CJ, Kim SR, Taskin O, Karakan I (1997): Sleep pattern changes in menstrual cycles of women with premenstrual syndrome: A preliminary study. Am J Obstet Gynecol 177:554-558.

Clare AW (1983): Psychiatric and social aspects of premenstrual complaints. Psychol Med Monogr Suppl 4:1-58.

Driver HS, Dijk DJ, Werth E (1996): Sleep and the sleep EEG across the menstrual cycle in young healthy women. $J$ Clin Endocrinol Metab 81:728-735.

Fisher DA, Odell WD (1971): Effect of cold on TSH secretion in man. J Clin Endocr Metab 33:859-862.

Hoddes E, Zarcone V, Smythe H, Phillips R, Dement WC (1973): Quantification of sleepiness: A new approach. Psychophysiology 10:431-436.

Ito M, Kohsaka M, Honma K, Fukuda N, Honma S, Katsuno Y, et al (1995): Changes of biological rhythm and sleep structure during the menstrual cycle in healthy women. Seishin Shinkeigaku Zasshi 97:155-164.

Kivelä A, Kauppila P, Ylostalo O, Vakkuri O, Leppaluoto J (1988): Seasonal, menstrual and circadian secretion of melatonin, gonadotropins and prolactin in women. Acta Physiol Scand 132:321-327.

Lack LC, Lushington K (1996): The rhythms of human sleep propensity and core body temperature. J Sleep Res 5:1-11.

Lavie P (1986): Ultrashort sleep-waking schedule. III "Gates" and "forbidden zones" for sleep. Electroencephalogr Clin Neurophysiol 63:414-425.

Lee KA (1988): Circadian temperature rhythms in relation to menstrual cycle phase. J Biol Rhythms 3:255-263.

Lewy AJ, Sack RL (1988): The dim light melatonin onset as a marker for circadian phase position. Chronobiol Int 6:93-102.
Mauri M, Reid RL, Maclean AW (1988): Sleep in premenstrual phase: A self-report study of PMS patients and normal controls. Acta Psychiatr Scand 78:82-86.

Minors DS, Waterhouse JM (1989): Masking in humans: The problem and some attempts to solve it. Chronobiol Int 6:29-53.

Nakagawa H, Sack RL, Lewy AJ (1992): Sleep propensity free-runs with the temperature, melatonin and cortisol rhythms in a totally blind person. Sleep 15:330-336.

Parry BL, Berga SL, Kripke DF, Klauber MR, Laughlin GA, Yen SSC, et al (1990): Altered waveform of plasma nocturnal melatonin secretion in premenstrual depression. Arch Gen Psychiatry 47:1139-1146.

Parry BL, Mendelson WB, Duncan WC, Sack DA, Wehr TA (1989): Longitudinal sleep EEG, temperature, and activity measurements across the menstrual cycle in patients with premenstrual depression and in age-matched controls. Psychiatry Res 30:285-303.

Rechtschaffen A, Kales A (1968): A Manual of Standardized Terminology, Techniques and Scoring System for Sleep Stages of Human Subjects. Washington, DC: U.S. Government Printing Office.

Reid RL (1985): Premenstrual syndrome. In: Leventhal JM, editor. Current Problems in Obstetrics, Gynecology and Fertility. Chicago: Year Book, 5-15.

Rodier WI (1971): Progesterone-estrogen interactions in the control of activity-wheel running in the female rat. J Comp Physiol Psychol 74:365-373.

Rogacz S, Duffy J, Czeisler CA (1987): Ambulatory body temperature monitoring in ovulatory women throughout MC. Sleep Res 16:633.

Sheldrake P, Cormack M (1976): Variation in menstrual cycle symptom reporting. J Psychosom Res 20:169-177.

Webley GE, Leidenberger F (1986): The circadian pattern of melatonin and its positive relationship with progesterone in women. J Clin Endocrinol Metab 63:323-328. 